\title{
Article \\ Synchronizability of Multi-Layer-Coupled Star-Composed Networks
}

\author{
Haiping Gao ${ }^{1}$, Jian $\mathrm{Zhu}^{2}$, Xianyong $\mathrm{Li}^{3}{ }^{3 * *}$ and Xing Chen ${ }^{2}$ \\ 1 Department of Basic Science, Xinjiang Institute of Light Industry Technology, Urumqi 830023, China; \\ yqh@xjie.edu.cn \\ 2 Department of Mathematics and Physics, Xinjiang Institute of Engineering, Urumqi 830023, China; \\ zj17@xjie.edu.cn (J.Z.); cx16@xjie.edu.cn (X.C.) \\ 3 School of Computer and Software Engineering, Xihua University, Chengdu 610039, China \\ * Correspondence: lixy@mail.xhu.edu.cn
}

check for

updates

Citation: Gao, H.; Zhu, J.; Li, X.; Chen, X. Synchronizability of Multi-Layer-Coupled Star-Composed Networks. Symmetry 2021, 13, 2224. https://doi.org/10.3390/sym13112224

Academic Editor: Lorentz Jäntschi

Received: 18 October 2021

Accepted: 17 November 2021

Published: 21 November 2021

Publisher's Note: MDPI stays neutral with regard to jurisdictional claims in published maps and institutional affiliations.

Copyright: (c) 2021 by the authors. Licensee MDPI, Basel, Switzerland. This article is an open access article distributed under the terms and conditions of the Creative Commons Attribution (CC BY) license (https:// creativecommons.org/licenses/by/ $4.0 /)$.

\begin{abstract}
In this paper, several multi-layer-coupled star-composed networks with similar symmetrical structures are defined by using the theory of graph operation. The supra-Laplacian matrix of the corresponding multi-layer networks is obtained according to the master stability equation (MSF). Two important indexes that reflect the synchronizability of these kinds of networks are derived in the case of bounded and unbounded synchronized regions. The relationships among the synchronizability, the number of layers, the length of the paths, the branchings, and the interlayer and intralayer coupling strengths in the two cases are studied. At the same time, the simulation experiments are carried out with the MATLAB software, and the simulated images of the two symmetrical structure networks' synchronizability are compared. Finally, the factors affecting the synchronizability of multi-layer-coupled star-composed networks are found. On this basis, optimization schemes are given to improve the synchronizability of multi-layer-coupled star-composed networks and the influences of the number of central nodes on the networks' synchronizability are further studied.
\end{abstract}

Keywords: interlayer coupling; star-composed networks; graph operation; synchronizability

\section{Introduction}

Complex networks have appeared in many aspects of our lives; they are used in the prevention of the spread of epidemic diseases, computer networks, social networks, and other fields [1-4]. These networks are an important interdisciplinary research field that has attracted the interest of many scholars. In recent years, many new advances have been made in the research of complex networks, such as the spread and stability of complex networks [5,6], the synchronization and consensus of multi-layer complex networks [7-15], diffusion in multi-layer networks [16-20], and the modeling and robustness of multi-layer complex networks [21-25].

Common network synchronization types are complete synchronization [8] and phase synchronization [26]. This paper studies the complete synchronization of networks according to the master stability equation (MSF) proposed by Pecora and Carroll [27]. Research on the synchronizability of multi-layer networks mostly focuses on structures such as star-like networks, and there has been little research on the synchronizability of multi-layer starcomposed networks. On the one hand, the symmetrical structures of multi-layer-coupled star-composed networks are more complex and the calculation of the synchronizability indexes in these symmetrical structures is more difficult than the calculation of the indexes of other types of structure. On the other hand, the characteristic polynomials of many star-composed coupled networks can not be solved analytically.

In recent years, scholars have obtained many classical results on the synchronization of star-like networks. Aguirre et al. [28] studied how the degree of nodes connected between two layers of networks affects the synchronizability of the whole system and proposed 
an effective synchronization strategy. Zhu et al. [29] studied the relationships among the synchronizability of two k-layer variable-coupling windmill-type networks, the number of nodes, the coupling strength, and other parameters in the case of unbounded and bounded synchronized regions and provided an optimization scheme to improve the synchronizability. Deng et al. [30] gave the characteristic polynomials of multi-layer-coupled chain networks, discussed the relationships between synchronizability and different parameters in the case of unbounded and bounded synchronized regions, and further provided image examples to verify the effectiveness of their theoretical analysis. Li et al. [31] studied the bipartite synchronization of coupled inertial memory neural networks with cooperative and competitive interaction. Two simulation examples were given to verify the effectiveness of the proposed discontinuous control strategy for bipartite synchronization. Inspired by the above works of literature, the main contents of this paper are as follows:

1. We provide definitions of star-composed networks and introduce the relevant theoretical knowledge required for the synchronization of multi-layer networks.

2. The supra-Laplacian matrix of multi-layer-coupled star-composed networks is given according to the MSF. With the help of graph theory and graph operation, the important indexes reflecting the synchronizability are obtained for the cases of bounded and unbounded synchronized regions.

3. According to the important indexes of synchronizability, we obtain the relationships between synchronizability and the parameters of multi-layer-coupled star-composed networks.

4. Through simulation experiments, we obtain the relationships between the synchronizability and various parameters, and provide a theoretical basis for optimizing the synchronizability of multi-layer-coupled star-composed networks.

The structure of this paper is as follows. In Section 2, the preliminaries required to study the synchronization of multi-layer-coupled star-composed networks are given. In Section 3, the eigenvalue spectrum and the synchronizability indexes of multi-layer-coupled star-composed networks are studied and extended to a more general case. In Section 4, the synchronizability of multi-layer-coupled star-composed networks is simulated numerically. In Section 5, the main conclusions of this paper are given.

\section{Preliminaries}

\subsection{The Synchronizability of Multi-Layer Networks}

According to the dynamic equation of the $i$ th node of the $L$ th layer in the $Q$-layer network [32]:

$$
\begin{gathered}
\frac{d x_{i}^{L}}{d t}=f\left(x_{i}^{L}\right)-a_{L} \sum_{j=1}^{N} \omega_{i j}^{L} \Phi\left(x_{j}^{L}\right)-d \sum_{T=1}^{Q} d_{i}^{L T} \mathrm{Y}\left(x_{i}^{L}\right), \\
i=1,2, \cdots, N, L=1,2, \cdots, Q .
\end{gathered}
$$

where $N$ is the number of nodes in each layer; $Q$ is the number of layers; $x_{i}^{L}$ is the state variable of the $i$ th node of the $L$ th layer; $f(*)$ is the dynamic function; $a_{L}$ is the intralayer coupling strength of the Lth layer; $d$ is the interlayer coupling strength; $\Phi$ is the intralayer coupling function; and $\mathrm{Y}$ is the interlayer coupling function. The property of the Laplacian matrix can ensure the block diagonalization of the Jacobian, which means that the MSF can be used to determine the stability of a synchronized solution by reducing the dimensionality of the synchronization problem [27], $H^{L}=\left(\omega_{i j}^{L}\right)=S^{L}-W^{L}$ is the Laplacian matrix of the Lth layer. Additionally, $S^{L}=\left(s_{i i}^{L}\right) \in R^{N \times N}$ is the degree matrix of the $L$ th layer, where $s_{i i}^{L}=d\left(v_{i}\right)$, and $d\left(v_{i}\right)$ is the degree of the vertex $v_{i} \cdot W^{L}=\left(w_{i j}^{L}\right) \in R^{N \times N}$ is the adjacency matrix of the $L$ th layer. If there is an edge between the vertex $v_{i}$ and $v_{j}$ of the Lth layer, $w_{i j}^{L}=1$; otherwise, $w_{i j}^{L}=0(i, j=1,2,3, \cdots, N-1, N)$.

In this paper, we consider the coupling strength of multi-layer networks. The weighted Laplacian matrix of the $L$ th layer is $H^{(L)}=a_{L}\left(S^{L}-W^{L}\right)$. The interlayer-weighted Laplacian matrix is defined as $d D=d\left(d_{i}^{L T}\right) \in R^{Q \times Q}(L, T=1,2,3, \cdots, Q)$. If there is an edge 
between the $i$ th node of the $L$ th layer and the $i$ th node of the $T$ th layer, $d_{i}^{L T}=-1$; otherwise, $d_{i}^{L T}=0$ and $d_{i}^{L L}=-\sum_{T=1, T \neq L}^{Q} d_{i}^{L T}(L, T=1,2,3, \cdots, Q)$.

The supra-Laplacian matrix of the multi-layer network is $H=H_{L}+H_{I}$, where $H_{I}=d D \otimes I_{N}, I_{N}$ is the identity matrix of $N \times N$, and $\otimes$ is the Kronecker product.

$$
H_{L}=\left(\begin{array}{ccccc}
H^{(1)} & 0 & \cdots & 0 & 0 \\
0 & H^{(2)} & \cdots & 0 & 0 \\
\vdots & \vdots & \vdots & \vdots & \vdots \\
0 & 0 & \cdots & H^{(Q-1)} & 0 \\
0 & 0 & \cdots & 0 & H^{(Q)}
\end{array}\right)=\bigoplus_{L=1}^{Q} H^{(L)}
$$

The eigenvalues of a square matrix $A$ are the roots of its characteristic polynomial $\Psi(\lambda)$, $\Psi(\lambda)=|\lambda I-A|$. The eigenvalues of matrices have many applications [8,33]. In this paper, the eigenvalues of the supra-Laplacian matrix $\mathrm{H}$ are used to study the synchronizability of the networks. The eigenvalues of the supra-Laplacian matrix $H$ are $\lambda_{1}, \lambda_{2}, \lambda_{3}, \cdots, \lambda_{n-1}, \lambda_{n}$. $\lambda_{1}=0$ is obtained from the properties of the supra-Laplacian matrix. According to the MSF, when the synchronized region is unbounded, the larger the $\lambda_{2}$ is, the stronger the synchronizability of the network is; when the synchronized region is bounded, the smaller the $r=\lambda_{\max } / \lambda_{2}$ is, the stronger the synchronizability of the network is [29].

\subsection{Graph Theory and Star-Composed Networks}

In this paper, we consider undirected and connected graph $G$. The vertex set of $G$ is $V(G)=\left\{v_{1}, v_{2}, v_{3}, \cdots, v_{n-1}, v_{n}\right\}$, and the edge set of $G$ is $E(G)=\left\{\left(v_{i}, v_{j}\right) \mid i, j=\right.$ $1,2,3 \cdots, n ; i \neq j\}$. The edge set and the vertex set uniquely determine the graph. Let $K_{p}$ be a complete graph with $p$ nodes, $P_{n}$ be a path of length $n$, and $E_{k}$ be a graph that has $k$ vertices with an empty edge set.

The star-composed networks studied in this paper are introduced below. We first give two graph operations.

Definition 1. ([34]) Let $G_{1}, G_{2}$ represent two simple graphs with $n_{1}, n_{2}$ vertices and $m_{1}, m_{2}$ edges, respectively. The corona operation $G_{1} \circ G_{2}$ is the graph obtained from one copy of $G_{1}$ and $n_{1}$ copies of $G_{2}$, before joining the ith vertex of $G_{1}$ to every vertex in the ith copy of $G_{2}\left(i=1,2, \cdots, n_{1}\right)$.

Definition 2. ([35]) Let $G_{1}, G_{2}$ represent two simple graphs. The conjunction operation $G_{1} \nabla G_{2}$ is defined as the graph, which is obtained from the disjoint union of $G_{1}$ and $G_{2}$ by adding the edges $\left\{a b: a \in V\left(G_{1}\right), b \in V\left(G_{2}\right)\right\}$.

$G_{1}(1, k, n)=\left(K_{1} \nabla E_{k}\right) \circ P_{n}$ is composed of one center node, $k$ branchings, and paths of length $n$. $G_{2}(2, k-1, n)=\left(K_{2} \nabla E_{k-1}\right) \circ P_{n}$ is composed of two center nodes, $k-1$ branchings, and paths of length $n$. Its connection relationships are shown in Figure 1 . The red nodes represent the central nodes, the yellow nodes represent the branchings nodes, the green nodes represent the nodes of the paths, and the black solid lines represent the interlayer connection relationships between the nodes.

$G_{1}^{Q}(1, k, n)$ and $G_{2}^{Q}(2, k-1, n)$ are composed of $Q$ layers $G_{1}(1, k, n)$ and $G_{2}(2, k-1, n)$, respectively. The total number of nodes is $Q(n k+n+k+1)$, and the connection relationships are shown in Figure 2. The colored dotted lines represent the connection relationships between each layer. 


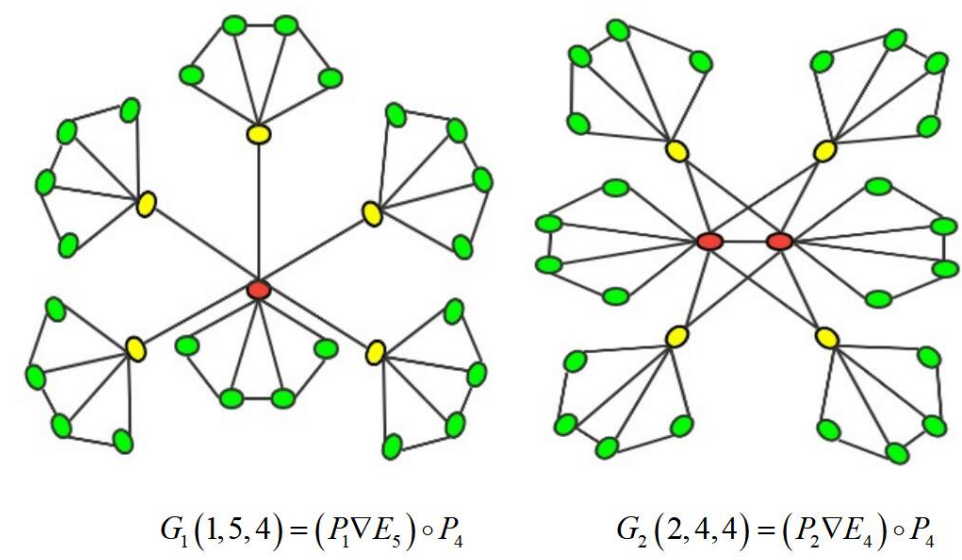

Figure 1. Single-layer star-composed network.
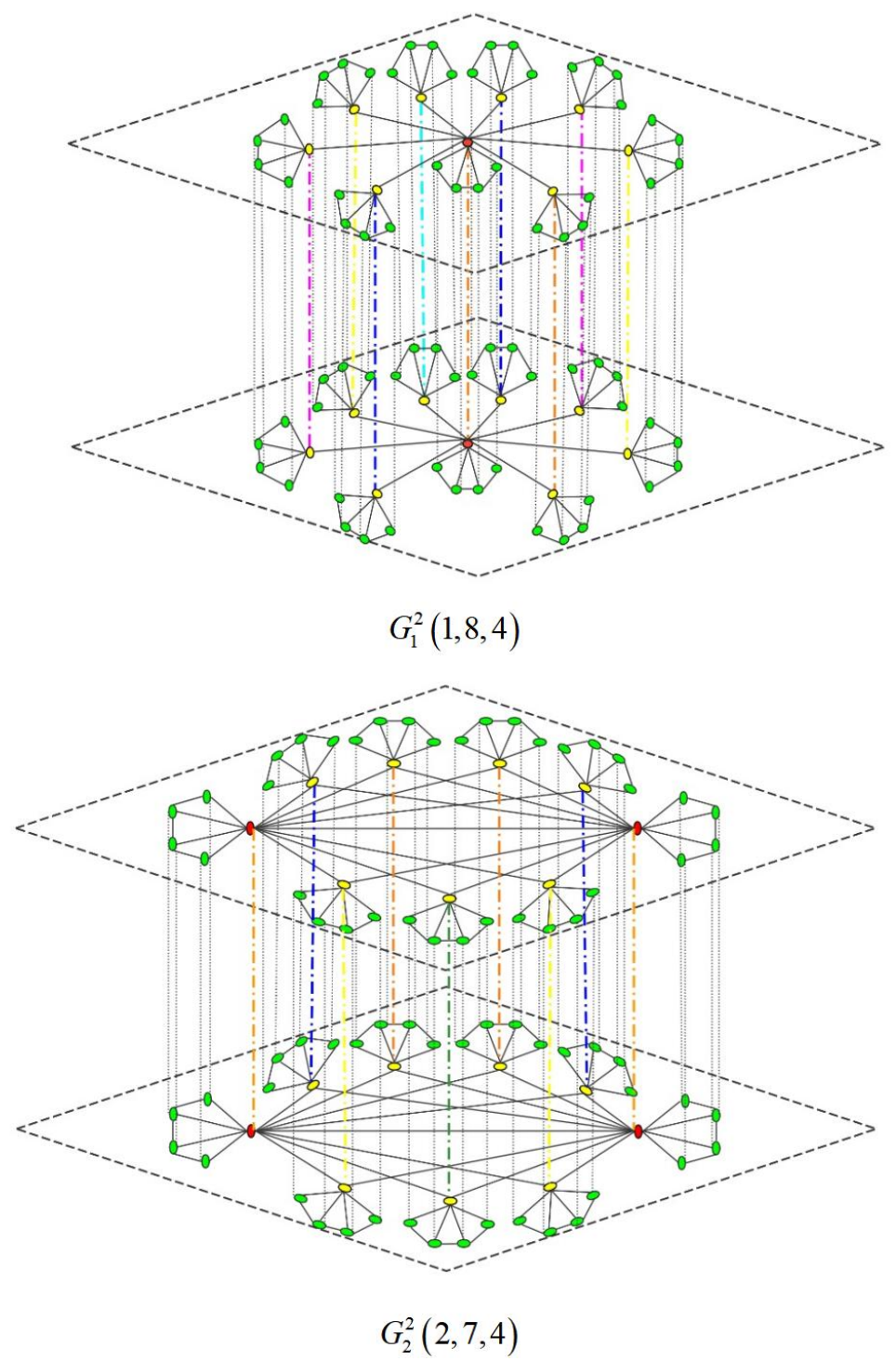

Figure 2. Double-layer star-composed network. 
Lemma 1. ([30]) Let $A, B$ be two $M \times M$ square matrices, and $K$ be an integer; then,

$$
\left|\begin{array}{cccc}
A & B & \cdots & B \\
B & A & \cdots & B \\
\vdots & \vdots & \ddots & \vdots \\
B & B & \cdots & A
\end{array}\right|_{K \times K}=|A+(K-1) B| \cdot|A-B|^{(K-1)}
$$

\section{The Eigenvalue Spectrum and Synchronizability Indexes of Multi-Layer Coupled} Star-Composed Networks

3.1. The Eigenvalue Spectrum and Synchronizability Indexes of $G_{1}^{Q}(1, k, n)$

Similar to the network structure of $G_{1}^{2}(1,8,4)$ in Figure 2, each layer has the same topology. We assume that $a$ is the intralayer coupling strength and $d$ is the interlayer coupling strength. According to the concept of supra-Laplacian matrix, it is not difficult to obtain the supra-Laplacian matrix of $G_{1}^{Q}(1, k, n)$ :

$$
H_{1}=\left(\begin{array}{ccccc}
(k+n) a & -a & -a & \cdots & -a \\
-a & \Theta_{1} & 0 & \cdots & 0 \\
-a & 0 & \Theta_{1} & \cdots & 0 \\
\vdots & \vdots & \vdots & \ddots & \vdots \\
-a & 0 & 0 & \cdots & \Theta_{1}
\end{array}\right)_{(k+1) \times(k+1)}
$$

where $\Theta_{1}=(n+1) a$.

$$
\begin{aligned}
H_{2}= & \left(\begin{array}{ccccc}
-a & 0 & 0 & \cdots & 0 \\
0 & -a & 0 & \cdots & 0 \\
0 & 0 & -a & \cdots & 0 \\
\vdots & \vdots & \vdots & \ddots & \vdots \\
0 & 0 & 0 & \cdots & -a
\end{array}\right)_{(k+1) \times(k+1)} \\
H_{3} & =\left(\begin{array}{ccccc}
2 a & 0 & 0 & \cdots & 0 \\
0 & 2 a & 0 & \cdots & 0 \\
0 & 0 & 2 a & \cdots & 0 \\
\vdots & \vdots & \vdots & \ddots & \vdots \\
0 & 0 & 0 & \cdots & 2 a
\end{array}\right)_{(k+1) \times(k+1)} \\
H_{4} & =\left(\begin{array}{ccccc}
3 a & 0 & 0 & \cdots & 0 \\
0 & 3 a & 0 & \cdots & 0 \\
0 & 0 & 3 a & \cdots & 0 \\
\vdots & \vdots & \vdots & \ddots & \vdots \\
0 & 0 & 0 & \cdots & 3 a
\end{array}\right)_{(k+1) \times(k+1)}
\end{aligned}
$$

The supra-Laplacian matrix of Lth layer is:

$$
H_{1}^{(L)}=\left(\begin{array}{ccccccc}
H_{1} & H_{2} & H_{2} & H_{2} & \cdots & H_{2} & H_{2} \\
H_{2} & H_{3} & H_{2} & 0 & \cdots & 0 & 0 \\
H_{2} & H_{2} & H_{4} & H_{2} & \cdots & 0 & 0 \\
H_{2} & 0 & H_{2} & H_{4} & \cdots & 0 & 0 \\
\vdots & \vdots & \vdots & \vdots & \ddots & \vdots & \vdots \\
H_{2} & 0 & 0 & 0 & \cdots & H_{4} & H_{2} \\
H_{2} & 0 & 0 & 0 & \cdots & H_{2} & H_{3}
\end{array}\right),
$$


Combined with the interlayer supra-Laplacian matrix:

$$
H=\left(\begin{array}{ccccc}
\Theta_{2} & -d I & -d I & \cdots & -d I \\
-d I & \Theta_{2} & -d I & \cdots & -d I \\
-d I & -d I & \Theta_{2} & \cdots & -d I \\
\vdots & \vdots & \vdots & \ddots & \vdots \\
-d I & -d I & -d I & \cdots & \Theta_{2}
\end{array}\right)
$$

where $\Theta_{2}=H_{1}^{(L)}+(Q-1) d I$.

Then, the characteristic polynomial is:

$$
\Psi_{I}(\lambda)=\operatorname{det}(\lambda I-H)=\left(\begin{array}{ccccc}
\Theta_{3} & d I & d I & \cdots & d I \\
d I & \Theta_{3} & d I & \cdots & d I \\
d I & d I & \Theta_{3} & \cdots & d I \\
\vdots & \vdots & \vdots & \ddots & \vdots \\
d I & d I & d I & \cdots & \Theta_{3}
\end{array}\right)
$$

where $\Theta_{3}=\lambda I-H_{1}^{(L)}-(Q-1) d I$.

According to Lemma 1, we have:

$$
\Psi_{I}(\lambda)=\left|\lambda I-H_{1}^{(L)}\right|\left|\lambda I-H_{1}^{(L)}-Q d I\right|^{Q-1} .
$$

The eigenvalue spectrum of $H$ is:

$$
\begin{aligned}
& 0,(n+1) a, \frac{k+n+2 \pm \sqrt{(k+n+2)^{2}-4(k+1)}}{2} a, \underbrace{\frac{n+2 \pm \sqrt{(n+2)^{2}-4}}{2} a}_{k-1}, \\
& \underbrace{Q d,(n+1) a+Q d}_{Q-1}, \underbrace{4 \operatorname{asin}^{2}\left(\frac{\alpha \pi}{2 n}\right)+a}_{k+1}(\alpha=1,2, \cdots, n-1), \\
& \underbrace{\frac{k+n+2 \pm \sqrt{(k+n+2)^{2}-4(k+1)}}{2} a+Q d}_{Q-1}, \\
& \underbrace{\frac{n+2 \pm \sqrt{(n+2)^{2}-4}}{2} a+Q d}_{(Q-1)(k-1)} \underbrace{4 \operatorname{asin}^{2}\left(\frac{\alpha \pi}{2 n}\right)+a+Q d}_{(Q-1)(k+1)}(\alpha=1,2, \cdots, n-1) .
\end{aligned}
$$

Deduced from the preliminary knowledge, the minimum nonzero eigenvalue is:

$$
\lambda_{2}=\min \left\{\frac{n+2-\sqrt{(n+2)^{2}-4}}{2} a, Q d\right\} .
$$

The maximum eigenvalue is:

$$
\lambda_{\max }=\frac{k+n+2+\sqrt{(k+n+2)^{2}-4(k+1)}}{2} a+Q d .
$$

Then, we have:

$$
r=\left(\frac{k+n+2+\sqrt{(k+n+2)^{2}-4(k+1)}}{2} a+Q d\right) / \lambda_{2} .
$$


3.2. The Eigenvalue Spectrum and Synchronizability Indexes of $G_{2}^{Q}(2, k-1, n)$

Similar to the network structure of $G_{2}^{2}(2,7,4)$ in Figure 2, we examine the eigenvalue spectrum of $G_{2}^{Q}(2, k-1, n)$ using a similar method:

$$
H_{5}=\left(\begin{array}{ccccc}
(k+n) a & -a & -a & \cdots & -a \\
-a & (k+n) a & -a & \cdots & -a \\
-a & -a & \Theta_{4} & \cdots & 0 \\
\vdots & \vdots & \vdots & \ddots & \vdots \\
-a & -a & 0 & \cdots & \Theta_{4}
\end{array}\right)_{(k+1) \times(k+1)}
$$

where $\Theta_{4}=(n+2) a$.

The supra-Laplacian matrix of the Lth layer is:

$$
H_{2}^{(L)}=\left(\begin{array}{ccccccc}
H_{5} & H_{2} & H_{2} & H_{2} & \cdots & H_{2} & H_{2} \\
H_{2} & H_{3} & H_{2} & 0 & \cdots & 0 & 0 \\
H_{2} & H_{2} & H_{4} & H_{2} & \cdots & 0 & 0 \\
H_{2} & 0 & H_{2} & H_{4} & \cdots & 0 & 0 \\
\vdots & \vdots & \vdots & \vdots & \ddots & \vdots & \vdots \\
H_{2} & 0 & 0 & 0 & \cdots & H_{4} & H_{2} \\
H_{2} & 0 & 0 & 0 & \cdots & H_{2} & H_{3}
\end{array}\right),
$$

Combined with the interlayer supra-Laplacian matrix:

$$
\tilde{H}=\left(\begin{array}{ccccc}
\Theta_{5} & -d I & -d I & \cdots & -d I \\
-d I & \Theta_{5} & -d I & \cdots & -d I \\
-d I & -d I & \Theta_{5} & \cdots & -d I \\
\vdots & \vdots & \vdots & \ddots & \vdots \\
-d I & -d I & -d I & \cdots & \Theta_{5}
\end{array}\right)
$$

where $\Theta_{5}=H_{2}^{(L)}+(Q-1) d I$.

The characteristic polynomial is:

$$
\Psi_{I I}(\lambda)=\left|\lambda I-H_{2}^{(L)}\right|\left|\lambda I-H_{2}^{(L)}-Q d I\right|^{Q-1} .
$$

The eigenvalue spectrum of $\tilde{H}$ is:

$$
\begin{aligned}
& 0,(n+1) a, \frac{k+n+2 \pm \sqrt{(k+n+2)^{2}-4(k+1)}}{2} a, \underbrace{\frac{n+3 \pm \sqrt{(n+3)^{2}-8}}{2} a}_{k-2}, \\
& \underbrace{Q d,(n+1) a+Q d}_{Q-1}, \underbrace{4 \operatorname{asin}^{2}\left(\frac{\beta \pi}{2 n}\right)+a}_{k+1}(\beta=1,2, \cdots, n-1), \\
& \underbrace{\frac{k+n+2 \pm \sqrt{(k+n+2)^{2}-4(k+1)}}{2} a+Q d}_{(Q-1)}, \\
& \underbrace{\frac{n+3 \pm \sqrt{(n+3)^{2}-8}}{2} a+Q d}_{(k-2)(Q-1)} \underbrace{4 \operatorname{asin}^{2}\left(\frac{\beta \pi}{2 n}\right)+a+Q d}_{(k+1)(Q-1)}(\beta=1,2, \cdots, n-1) .
\end{aligned}
$$

By comparison, the minimum nonzero eigenvalue is:

$$
\lambda_{2}=\min \left\{\frac{n+3-\sqrt{(n+3)^{2}-8}}{2} a, Q d\right\} .
$$


The maximum eigenvalue is:

$$
\lambda_{\max }=\frac{k+n+2+\sqrt{(k+n+2)^{2}-4(k+1)}}{2} a+Q d .
$$

Then, we have:

$$
r=\left(\frac{k+n+2+\sqrt{(k+n+2)^{2}-4(k+1)}}{2} a+Q d\right) / \lambda_{2}
$$

3.3. The Eigenvalue Spectrum and Synchronizability Indexes of $G_{p}^{Q}(p, k+1-p, n)$

The multi-layer-coupled star-composed networks with $p$ central nodes are considered, ( $3 \leq p \leq k)$, and the multi-center-coupled star-composed networks can be written as $G_{p}^{Q}(p, k+1-p, n)=\left(K_{p} \nabla E_{k+1-p}\right) \circ P_{n}$.

The eigenvalue spectrum of the supra-Laplace matrix obtained by the same method is:

$0,(n+1) a, \underbrace{\frac{k+n+2 \pm \sqrt{(k+n+2)^{2}-4(k+1)}}{2}}_{p} a \underbrace{\frac{n+p+1 \pm \sqrt{(n+p+1)^{2}-4 p}}{2}}_{k-p} a$,

$$
\underbrace{Q d,(n+1) a+Q d}_{Q-1}, \underbrace{4 \operatorname{asin}^{2}\left(\frac{\gamma \pi}{2 n}\right)+a}_{k+1}(\gamma=1,2, \cdots, n-1),
$$

$$
\underbrace{\frac{k+n+2 \pm \sqrt{(k+n+2)^{2}-4(k+1)}}{2} a+Q d}_{p(Q-1)},
$$

$$
\underbrace{\frac{n+p+1 \pm \sqrt{(n+p+1)^{2}-4 p}}{2} a+Q d}_{(k-p)(Q-1)}, \underbrace{4 \operatorname{asin}^{2}\left(\frac{\gamma \pi}{2 n}\right)+a+Q d}_{(k+1)(Q-1)}(\gamma=1,2, \cdots, n-1) .
$$

We can easily obtain the required indicators. The minimum nonzero eigenvalue is as follows:

$$
\lambda_{2}=\min \left\{\frac{n+p+1-\sqrt{(n+p+1)^{2}-4 p}}{2} a, Q d\right\} .
$$

The maximum eigenvalue is:

$$
\lambda_{\max }=\frac{k+n+2+\sqrt{(k+n+2)^{2}-4(k+1)}}{2} a+Q d .
$$

Then, we have:

$$
r=\left(\frac{k+n+2+\sqrt{(k+n+2)^{2}-4(k+1)}}{2} a+Q d\right) / \lambda_{2}
$$

\section{Numerical Simulation Experiment and Analysis}

In the analysis of the above sections, we can obtain the relationships between the synchronizability and various parameters of multi-layer-coupled star-composed networks. This section tests the accuracy of the above mentioned theoretical results through a large number of numerical simulation experiments. Firstly, the state trajectories of star-composed networks are described to illustrate that their synchronization can be realized by MATLAB. Secondly, considering the actual initial value, the change images of the synchronizability of the starcomposed networks are obtained by MATLAB. Finally, we analyze the relationships between the synchronizability and parameters of multi-layer-coupled star-composed networks. 
We consider an example of a coupling system composed of two layers. The network topology is shown in Figure 3. The number of layers is $Q=2, N=9$ is the number of nodes in each layer, and the dynamics of each node are given as follows [36]:

$$
\frac{d x_{i}^{L}}{d t}=\frac{\left|x_{i}^{L}+1\right|-\left|x_{i}^{L}-1\right|}{2}+\sum_{j \in N_{i}} a_{i j}\left(x_{j}^{L}-x_{i}^{L}\right)+\sum_{j \in N_{i}} b_{i j}\left(x_{j}^{3-L}-x_{i}^{L}\right)
$$

where $x_{i}^{L}$ is the state of a node, $\frac{|x+1|-|x-1|}{2}$ represents the inherent nonlinear dynamics of the node, $i=1,2,3, \cdots, 8,9, L=1,2 ; a_{i j}$ represents the intralayer connection, and the connection weight is $1 . b_{i j}$ denotes the interlayer connection, and the connection weight is 2.5. The initial value of the system is randomly selected. Through the simulation, the state trajectory of the system is shown in Figures 4 and 5. It can be seen that the state trajectory of nodes achieves synchronization under the coupling effect.

In the following analysis, in order to distinguish the synchronizability indexes of multilayer-coupled star-composed networks, when the synchronized region is unbounded, we use $\lambda_{2 a}$ and $\lambda_{2 b}$ to represent the synchronizability indexes of $G_{1}^{Q}(1, k, n)$ and $G_{2}^{Q}(2, k-1, n)$. When the synchronized region is bounded, the synchronizability indexes of $G_{1}^{Q}(1, k, n)$ and $G_{2}^{Q}(2, k-1, n)$ are represented by $r_{a}$ and $r_{b}$, respectively.

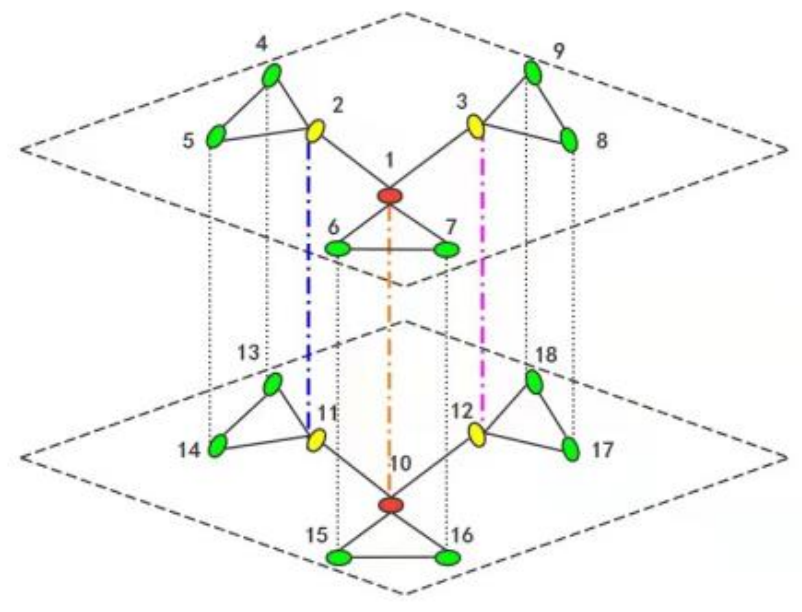

Figure 3. An example of double-layer star-composed network.

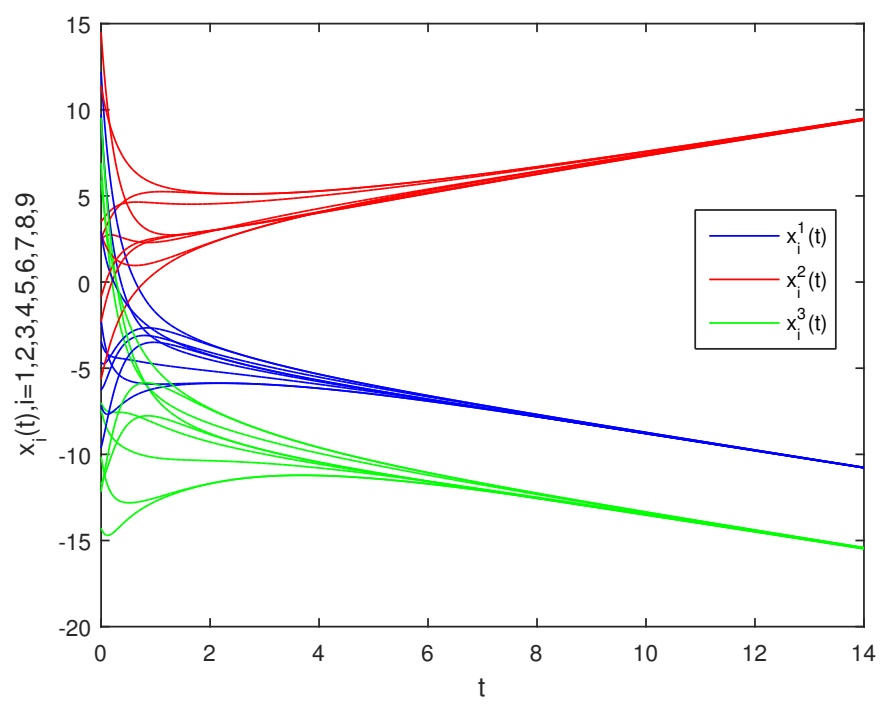

Figure 4. The state trajectories of the first layer. 


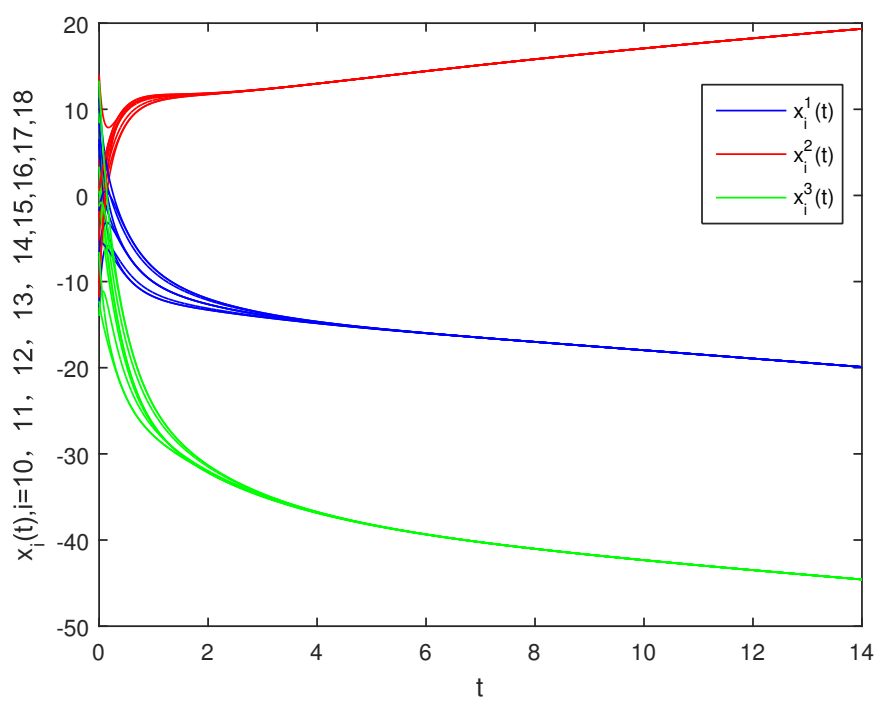

Figure 5. The state trajectories of the second layer.

1. When the synchronized region is unbounded, we set $n=5, a=2, d=0.008, Q=40$, with the increase in $k$ as in Figure 6. $\lambda_{2 a}$ remains unchanged at 0.2918 and $\lambda_{2 b}$ remains unchanged at 0.32 . The synchronizability is not affected by $k$. We set $k=10, a=2$, $d=0.008, Q=40$. With the increase in $n$ in Figure $7, \lambda_{2 a}$ remains unchanged at 0.32 and then decreases monotonically to 0.0196 , while $\lambda_{2 b}$ remains unchanged at 0.32 and then decreases monotonically to 0.0486 . The synchronizability of $G_{1}^{Q}(1, k, n)$ and $G_{2}^{Q}(2, k-1, n)$ reached the maximum at $n=\lfloor a / Q d+Q d / a-2\rfloor$ and $n=\lfloor 2 a / Q d+Q d / a-3\rfloor$, respectively, and the length of the paths reaches the maximum. We set $k=10, n=5, d=0.008, Q=40$, with the increase in $a$ as in Figure 8. $\lambda_{2 a}$ monotonically increases from 0.0729 to 0.32 and then remains unchanged, while $\lambda_{2 b}$ monotonically increases from 0.1629 to 0.32 and then remains unchanged. The synchronizability of $G_{1}^{Q}(1, k, n)$ and $G_{2}^{Q}(2, k-1, n)$ reaches the maximum at $a=\left(n+2+\sqrt{(n+2)^{2}-4}\right) Q d / 2$ and $a=\left(n+3+\sqrt{(n+3)^{2}-8}\right) Q d / 4$, respectively, and the intralayer coupling strength is the minimum. We set $k=10, n=5$, $a=2, Q=40$. With the increase in $d$ in Figure $9, \lambda_{2 a}$ monotonically increases from 0.04 to 0.2918 and then remains unchanged, while $\lambda_{2 b}$ monotonically increases from 0.04 to 0.6515 and then remains unchanged. The synchronizability of $G_{1}^{Q}(1, k, n)$ and $G_{2}^{Q}(2, k-1, n)$ reaches the maximum at $d=\left(n+2-\sqrt{(n+2)^{2}-4}\right) a / 2 Q$ and $d=\left(n+3-\sqrt{(n+3)^{2}-8}\right) a / 2 Q$, respectively, and the interlayer coupling strength is the minimum. We set $k=10, n=5, a=2, d=0.008$, with the increase in as $Q$ in Figure 10. $\lambda_{2 a}$ monotonically increases from 0.016 to 0.2918 and then remains unchanged, while $\lambda_{2 b}$ monotonically increases from 0.016 to 0.6515 and then remains unchanged. The synchronizability of $G_{1}^{Q}(1, k, n)$ and $G_{2}^{Q}(2, k-1, n)$ reaches the maximum at $Q=\left(n+2-\sqrt{(n+2)^{2}-4}\right) a / 2 d$ and $Q=\left(n+3-\sqrt{(n+3)^{2}-8}\right) a / 2 d$, respectively, and the number of layers is the minimum.

2. When the synchronized region is bounded, we set $n=5, a=2, d=0.008, Q=40$, with the increase in $k$ as in Figure 6. $r_{a}$ monotonically increases from 91.2508 to 1094.7 and $r_{b}$ monotonically increases from 83.2082 to 998.1725 . When $k$ is the minimum, the synchronizability is the maximum. We set $k=10, a=2, d=0.008, Q=40$. With the increase in $n$ in Figure 7, $r_{a}$ rapidly increases from 127.0276 to 17141 and $r_{b}$ rapidly increases from 127.0276 to 6922.5 . When $n$ is the minimum, the synchronizability is the maximum. We set $k=10, n=5, d=0.008, Q=40$. With the increase in $a$ in Figure 8, $r_{a}$ decreases slowly from 174.5482 to 171.1677 and then increases rapidly to 233.7460 , while $r_{b}$ decreases slowly from 78.1736 to 77.2113 and then 
increases rapidly to 233.7460. The synchronizability of $G_{1}^{Q}(1, k, n)$ and $G_{2}^{Q}(2, k-$ $1, n)$ reaches the maximum at $a=\left(n+2+\sqrt{(n+2)^{2}-4}\right) Q d / 2$ and $a=(n+3+$ $\left.\sqrt{(n+3)^{2}-8}\right) Q d / 4$, respectively. We set $k=10, n=5, a=2, Q=40$. With the increase in $d$ in Figure 9, $r_{a}$ decreases rapidly from 1242.3 to 171.2582 and then increases monotonically to 581.4077 , while $r_{b}$ decreases slowly from 1242.3 to 77.2526 and then increases rapidly to 260.3906 . The synchronizability of $G_{1}^{Q}(1, k, n)$ and $G_{2}^{Q}(2, k-1, n)$ reaches the maximum at $d=\left(n+2-\sqrt{(n+2)^{2}-4}\right) a / 2 Q$ and $d=$ $\left(n+3-\sqrt{(n+3)^{2}-8}\right) a / 2 Q$, respectively. We set $k=10, n=5, a=2, d=0.008$. With the increase in $Q$ in Figure 10, $r_{a}$ rapidly decreases from 3104.3 to 171.1760 and then slowly increases to 172.9032 , while $r_{b}$ decreases rapidly from 3104.3 to 77.2158 and then increases slowly to 77.4368. The synchronizability of $G_{1}^{Q}(1, k, n)$ and $G_{2}^{Q}(2, k-1, n)$ reaches the maximum at $Q=\left(n+2-\sqrt{(n+2)^{2}-4}\right) a / 2 d$ and $Q=\left(n+3-\sqrt{\left.(n+3)^{2}-8\right)} a / 2 d\right.$, respectively.
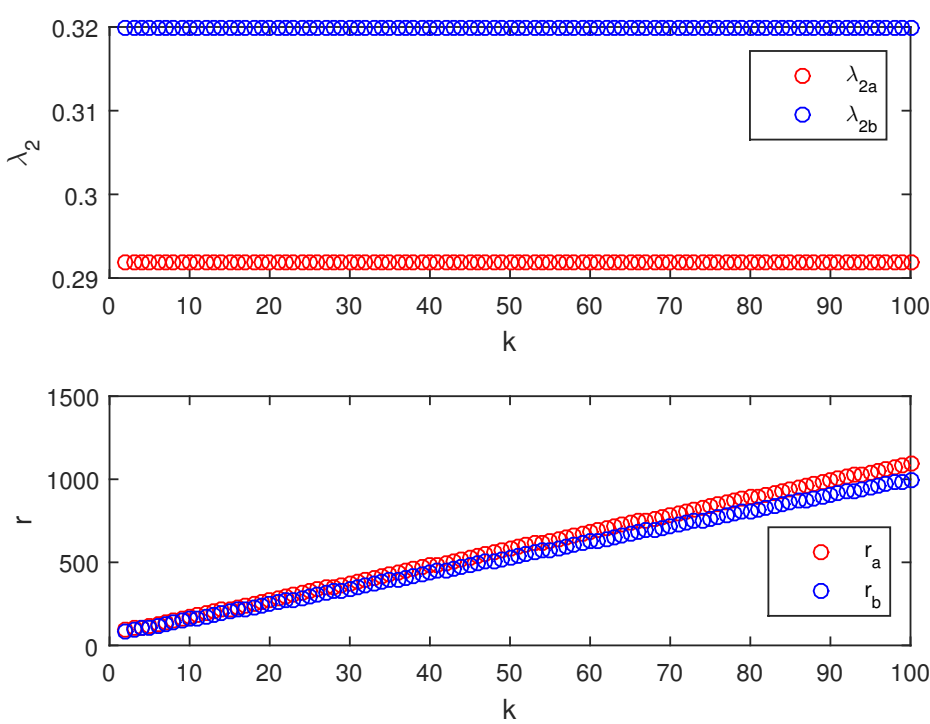

Figure 6. $\lambda_{2}$ and $r$ change with $k$.
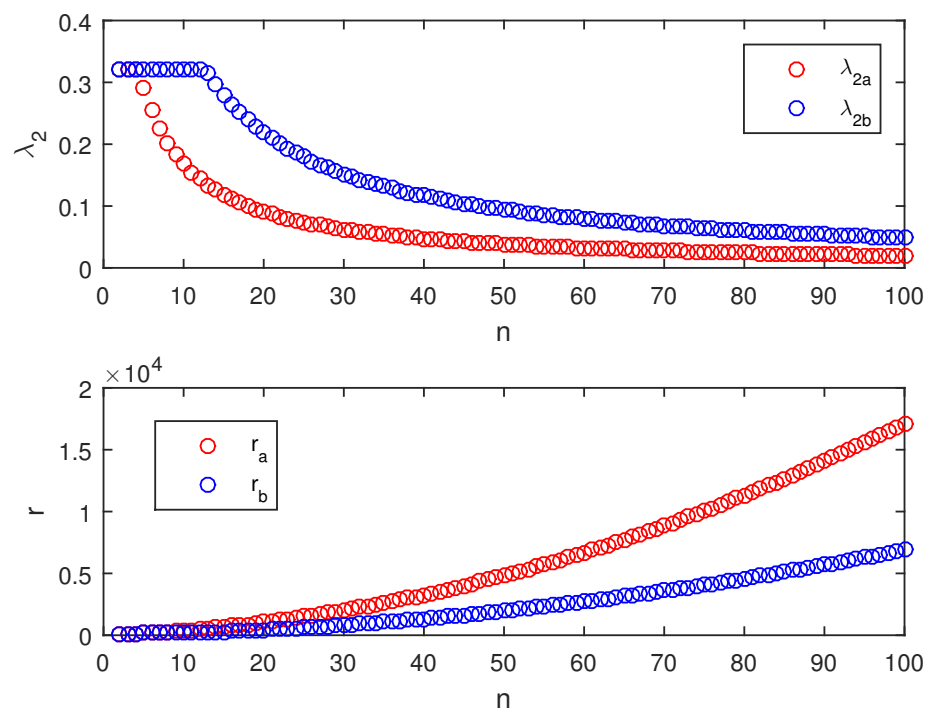

Figure 7. $\lambda_{2}$ and $r$ change with $n$. 

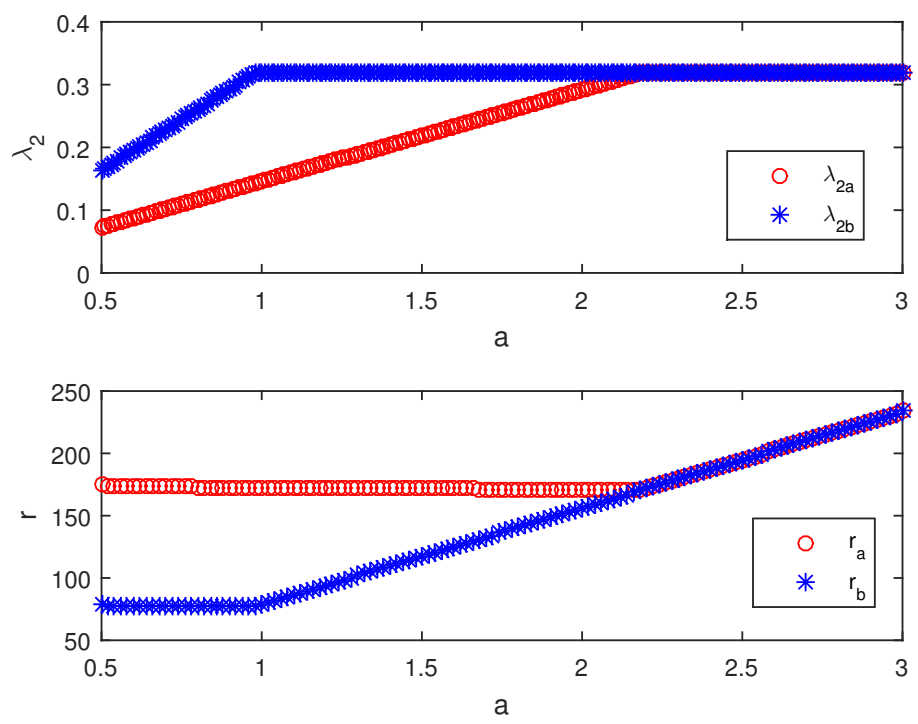

Figure 8. $\lambda_{2}$ and $r$ change with $a$.
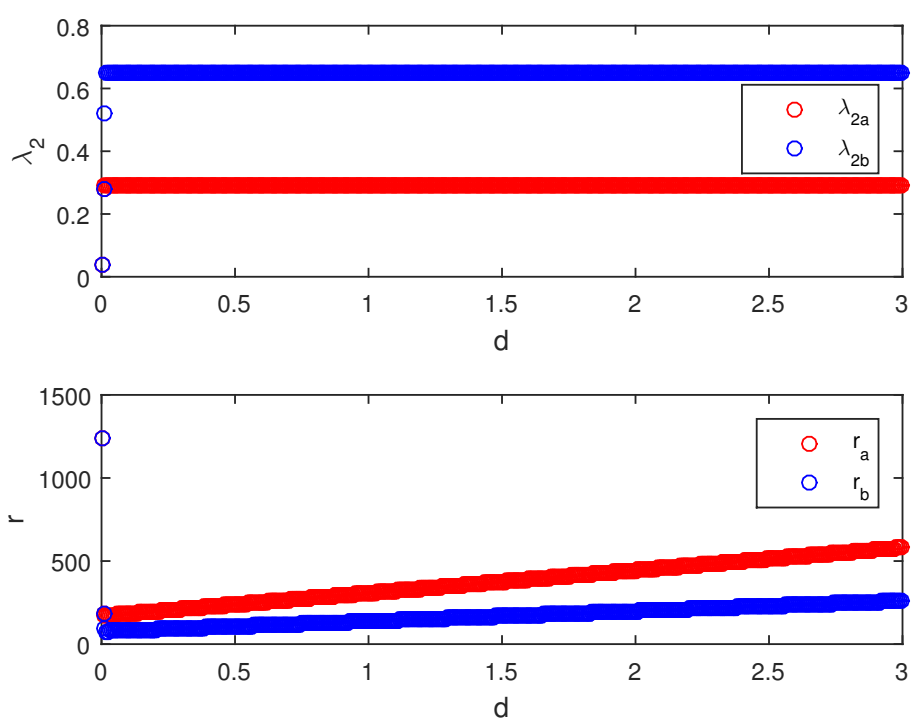

Figure 9. $\lambda_{2}$ and $r$ change with $d$. 

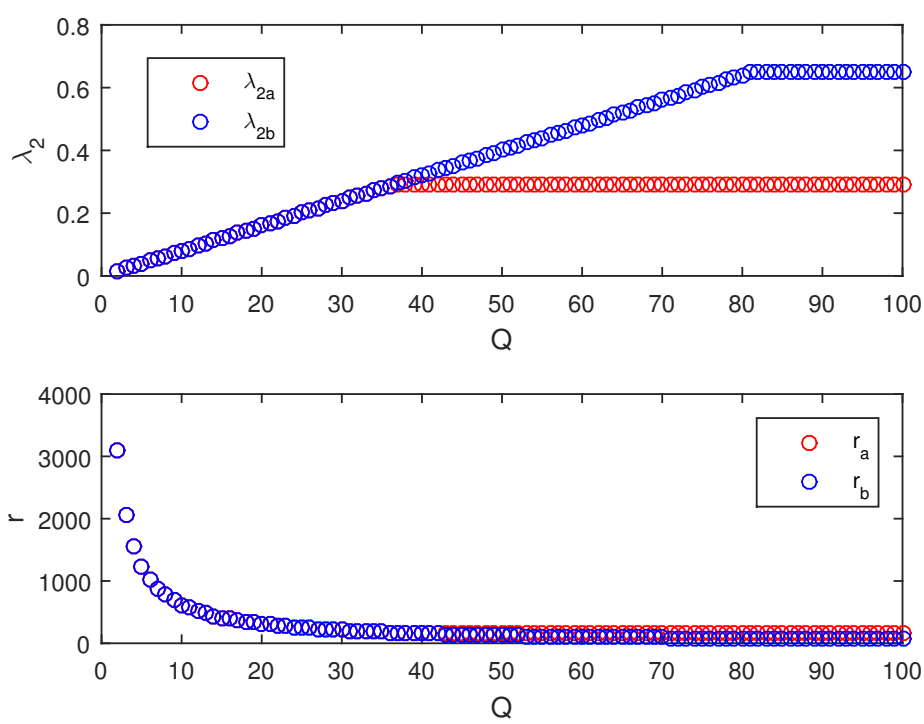

Figure 10. $\lambda_{2}$ and $r$ change with $Q$.

\section{Main Results}

\subsection{The Parameters That Affect the Synchronizability of Star-Composed Networks}

Based on the above situation, for $G_{1}^{Q}(1, k, n)$ networks when the synchronized region is unbounded, the synchronizability of the networks is only related to the number of layers, the length of paths, the interlayer coupling strength, and the intralayer coupling strength. When the synchronized region is bounded, the synchronizability of the networks is related to the interlayer coupling strength, the intralayer coupling strength, the length of paths, the branchings, and the number of layers. See Table 1 for the specific relationships. For $G_{2}^{Q}(2, k-1, n)$ networks, whether the synchronized region is bounded or unbounded, the factors affecting the networks' synchronizability are the same as $G_{1}^{Q}(1, k, n)$; see Table 2 for the specific relationships.

Table 1. Relationships among all parameters affecting $G_{1}^{Q}(1, k, n)$ networks' synchronizability.

\begin{tabular}{ccccccc}
\hline & & $k \uparrow$ & $n \uparrow$ & $a \uparrow$ & $d \uparrow$ & $Q \uparrow$ \\
\hline \multirow{2}{*}{$\lambda_{2}=\min \left\{Q d, \Delta_{1}\right\}$} & $\Delta_{1}<Q d$ & - & $\downarrow$ & $\uparrow$ & - & - \\
\cline { 2 - 6 } & $\Delta_{1}>Q d$ & - & - & - & $\uparrow$ & $\uparrow$ \\
\hline \multirow{2}{*}{$r=\lambda_{\max } / \lambda_{2}$} & $\Delta_{1}<Q d$ & $\uparrow$ & $\uparrow$ & $\downarrow$ & $\uparrow$ & $\uparrow$ \\
\cline { 2 - 6 } & $\Delta_{1}>Q d$ & $\uparrow$ & $\uparrow$ & $\uparrow$ & $\downarrow$ & $\downarrow$ \\
\hline
\end{tabular}

$\Delta_{1}=\frac{n+2-\sqrt{(n+2)^{2}-4}}{2} a, \uparrow$ strengthen, $\downarrow$ weaken, - unchanged.

Table 2. Relationships among all parameters affecting $G_{2}^{Q}(2, k-1, n)$ networks' synchronizability.

\begin{tabular}{ccccccc}
\hline & & $k \uparrow$ & $n \uparrow$ & $a \uparrow$ & $d \uparrow$ & $Q \uparrow$ \\
\hline \multirow{2}{*}{$\lambda_{2}=\min \left\{Q d, \Delta_{2}\right\}$} & $\Delta_{2}<Q d$ & - & $\downarrow$ & $\uparrow$ & - & - \\
\cline { 2 - 6 } & $\Delta_{2}>Q d$ & - & - & - & $\uparrow$ & $\uparrow$ \\
\hline \multirow{2}{*}{$r=\lambda_{\max } / \lambda_{2}$} & $\Delta_{2}<Q d$ & $\uparrow$ & $\uparrow$ & $\downarrow$ & $\uparrow$ & $\uparrow$ \\
\cline { 2 - 6 } & $\Delta_{2}>Q d$ & $\uparrow$ & $\uparrow$ & $\uparrow$ & $\downarrow$ & $\downarrow$ \\
\hline
\end{tabular}

$\overline{\Delta_{2}=\frac{n+3-\sqrt{(n+3)^{2}-8}}{2}} a, \uparrow$ strengthen, $\downarrow$ weaken, - unchanged. 


\subsection{Optimizing Synchronizability of $G_{p}^{Q}(p, k+1-p, n)$ Networks}

We further discuss the synchronizability of the multi-layer-coupled star-composed networks with the central nodes $p$. Figures $6-10$ show the relationships between the synchronizability of $G_{p}^{Q}(p, k+1-p, n)(p=1,2)$ and the affecting factors. $G_{p}^{Q}(p, k+1-$ $p, n)$ have similar figures; the specific relationship is shown in Table 3.

Table 3. Relationships between all parameters affecting $G_{p}^{Q}(p, k+1-p, n)(3 \leq p \leq k)$ networks synchronizability.

\begin{tabular}{ccccccc}
\hline & & $k \uparrow$ & $n \uparrow$ & $a \uparrow$ & $d \uparrow$ & $Q \uparrow$ \\
\hline \multirow{2}{*}{$\lambda_{2}=\min \left\{Q d, \Delta_{3}\right\}$} & $\Delta_{3}<Q d$ & - & $\downarrow$ & $\uparrow$ & - & - \\
\cline { 2 - 6 } & $\Delta_{3}>Q d$ & - & - & - & $\uparrow$ & $\uparrow$ \\
\hline \multirow{2}{*}{$r=\lambda_{\max } / \lambda_{2}$} & $\Delta_{3}<Q d$ & $\uparrow$ & $\uparrow$ & $\downarrow$ & $\uparrow$ & $\uparrow$ \\
\cline { 2 - 6 } & $\Delta_{3}>Q d$ & $\uparrow$ & $\uparrow$ & $\uparrow$ & $\downarrow$ & $\downarrow$ \\
\hline
\end{tabular}

$\Delta_{3}=\frac{n+p+1-\sqrt{(n+p+1)^{2}-4 p}}{2} a, \uparrow$ strengthen, $\downarrow$ weaken, - unchanged.

1. When the synchronized region is unbounded, the synchronizability is not affected by $k$. With the increase in the length of paths $n$, the synchronizability of the networks remains unchanged and then decreases. The synchronizability of $G_{p}^{Q}(p, k+1-p, n)$ reaches the maximum at $n=\lfloor a p / Q d+Q d / a-(p+1)\rfloor$ and the length of paths reaches the maximum. With the increase in intralayer coupling strength $a$, the synchronizability of the networks increases first and then remains unchanged. The synchronizability of $G_{p}^{Q}(p, k+1-p, n)$ reaches the maximum at $a=(n+p+1+$ $\left.\sqrt{(n+p+1)^{2}-4 p}\right) Q d / 2 p$, and the intralayer coupling strength is the minimum. With the increase in interlayer coupling strength $d$, the synchronizability of the networks increases first and then remains unchanged. The synchronizability of $G_{p}^{Q}(p, k+1-p, n)$ reaches the maximum at $d=\left(n+p+1-\sqrt{(n+p+1)^{2}-4 p}\right) a / 2 Q$ and the interlayer coupling strength is the minimum. With the increase in the number of layers $Q$, the synchronizability of the networks increases first and then remains unchanged. The synchronizability of $G_{p}^{Q}(p, k+1-p, n)$ reaches the maximum at $Q=$ $\left(n+p+1-\sqrt{(n+p+1)^{2}-4 p}\right) a / 2 d$ and the number of layers is the minimum. It can be seen that in order to improve the synchronizability of the networks, the length of paths $n$ should be appropriately reduced, and the intralayer coupling strength $a$, interlayer coupling strength $d$, and number of layers $Q$ should be appropriately increased.

2. When the synchronized region is bounded, with the increase in the branchings $k$ and the length of paths $n$, the synchronizability of the networks continues to weaken. With the increase in the intralayer coupling strength $a$, the synchronizability of the networks increases first and then decreases. The synchronizability of $G_{p}^{Q}(p, k+1-p, n)$ reaches the maximum at $a=\left(n+p+1+\sqrt{\left.(n+p+1)^{2}-4 p\right)}\right) d / 2 p$. With the increase in the interlayer coupling strength $d$, the synchronizability of the networks increases first and then decreases. The synchronizability of $G_{p}^{Q}(p, k+1-p, n)$ reaches the maximum at $d=\left(n+p+1-\sqrt{(n+p+1)^{2}-4 p}\right) a / 2 Q$. With the increase in the number of layers $Q$, the synchronizability of the networks increases first and then decreases. The synchronizability of $G_{p}^{Q}(p, k+1-p, n)$ reaches the maximum at $Q=\left(n+p+1-\sqrt{(n+p+1)^{2}-4 p}\right) a / 2 d$. From the above analysis, in order to improve the synchronizability of the networks, we reduce the branchings $k$ and the length of paths $n$ and appropriately increase the intralayer coupling strength $a$, interlayer coupling strength $d$, and number of layers $Q$ to the corresponding inflection points. 


\subsection{Impact of the Number of Central Nodes on the Synchronizability}

In the numerical simulation, we determined that the synchronizability of the networks with two central nodes is better than that of the networks with one central node. Whether the synchronized region is bounded or unbounded, with the increase in the central nodes $p$, the synchronizability of the networks will not be weaker than the synchronizability of the networks with fewer central nodes.

There are still many problems that need to be solved in multi-layer-coupled starcomposed networks. For example, what will happen to the synchronizability after giving different coupling strengths to multi-layer-coupled star-composed networks? For multi-layer-coupled star-composed networks, we need to further study how to keep the synchronizability unchanged when changing multiple parameters.

Author Contributions: Conceptualization, H.G. and J.Z.; methodology, H.G. and J.Z.; software, H.G. and J.Z.; validation, H.G., J.Z., and X.C.; formal analysis, H.G. and X.C.; writing-original draft preparation, H.G. and J.Z.; writing-review and editing, X.L.; supervision, X.L. and X.C.; project administration, H.G. All authors have read and agreed to the published version of the manuscript.

Funding: This work was supported by the National Natural Science Foundation of China (No. 61802316) and the National Science Foundation of Xinjiang (NSFXJ), (No. 2021D01A65), the Scientific Research and Education Project of Xinjiang Institute of Engineering, (No. 2020xgy332302), and the National innovation and entrepreneurship training program for College Students (No. 202110994006).

Institutional Review Board Statement: Not applicable.

Informed Consent Statement: Not applicable.

Data Availability Statement: Some or all of the data, models, or code generated or used during the study are available from the corresponding author by request.

Acknowledgments: We express our sincere gratitude to the people who gave us valuable comments.

Conflicts of Interest: The authors declare no conflict of interest.

\section{References}

1. Granell, C.; Gómez, S.; Arenas, A. Dynamical interplay between awareness and epidemic spreading in multiplex networks. Phys. Rev. Lett. 2013, 111, 128701. [CrossRef] [PubMed]

2. Min, B.; Yi, S.; Lee, K.; Goh, K. Network robustness of multiplex networks with interlayer degree correlations. Phys. Rev. E 2014, 89, 042811. [CrossRef] [PubMed]

3. Borgatti, S.; Mehra, A.; Brass, D.; Labianca, G.; Labianca, G. Network analysis in the social sciences. Science 2009, 323, $892-895$. [CrossRef]

4. Huang, S.; Zhang, J.; Wang, L.; Hu, X. Social friend recommendation based on multiple network correlation. IEEE Trans. Multimed. 2016, 18, 287-299. [CrossRef]

5. Ramos, L.A.A.; Jaquez, R.B.; Schaum, A. Output-Feedback Control for Discrete-Time Spreading Models in Complex Networks. Entropy 2018, 20, 204. [CrossRef]

6. Jaquez, R.B.; Ramos, L.A.A.; Schaum, A. Spreading Control in Two-Layer Multiplex Networks. Entropy 2020, 22, 1157. [CrossRef]

7. Jalan, S.; Kumar, A.; Schaum, I.L. Explosive synchronization in frequency displaced multiplex networks. Chaos 2019, $29,041102$. [CrossRef]

8. Li, J.; Luan, Y.; Wu, X.; Lu, J. Synchronizability of double-layer dumbbell net-works. Chaos 2021, 31, 073101. [CrossRef]

9. Zhang, H.; Ye, M.; Ye, R.; Cao, J. Synchronization stability of Riemann-Liouville fractional delay-coupled complex neural networks. Phys. A Stat. Mech. Its Appl. 2018, 508, 155-165. [CrossRef]

10. Xu, M.; Lu, J.; Zhou, J. Synchronizability and eigenvalues of two-layer star networks. Acta Phys. Sin. 2016, 65, 028902.

11. Huang, D.; Zhu, J.; Yu, Z.; Jiang, H. Cluster-delay consensus in MASS with layered intermittent communication: A multi-tracking approach. Symmetry 2021, 13, 1248. [CrossRef]

12. Sun, W.; Li, Y.; Liu, S. Noisy consensus dynamics in windmill-type graphs. Chaos 2020, 30, 123131. [CrossRef] [PubMed]

13. Zhou, M.; Li, X.; Xiong, W.; Wu, X.; Liao, H. Cooperation of local and collective synchronization in complex networks. Phys. A Stat. Mech. Its Appl. 2019, 526, 120963. [CrossRef]

14. Vaseghi, B.; Mobayen, S.; Hashemi, S.S.; Fekih, A. Fast reaching fifinite time synchronization approach for chaotic systems with application in medical image encryption. IEEE Access 2021, 9, 25911-25925. [CrossRef]

15. Hu, C.; He, H.; Jiang, H. Synchronization of complex-valued dynamic networks with intermittently adaptive coupling: a direct error method. Automatica 2019, 112, 108675. [CrossRef] 
16. Kan, J.; Ma, C.; Zhang, H.; Xiang, B. Interplay of epidemic spreading and strategy-mixed awareness diffusion on multiplex networks. Int. J. Mod. Phys. C 2020, 31, 2050085. [CrossRef]

17. Niu, R.; Wu, X.; Lu, J.; Lü, J. Adaptive Diffusion Processes of Time-Varying Local Information on Networks. IEEE Trans. Circuits Syst. II Express Briefs 2019, 66, 1592-1596. [CrossRef]

18. Yu, Q.; Yu, Z.; Ma, D. A Multiplex Network Perspective of Innovation Diffusion: An Information-Behavior Framework. IEEE Access 2020, 8, 36427-36440. [CrossRef]

19. Wang, X.; Tejedor, A.; Wang, Y.; Moreno, Y. Unique superdiffusion induced by directionality in multiplex networks. New J. Phys. 2021, 23, 013016. [CrossRef]

20. Yan, H.; Zhou, J.; Li, W.; Lu, J.; Fan, R. Superdiffusion criteria on duplex networks. Chaos 2021, 31, 073108. [CrossRef]

21. Sun, W.; Sun, M.; Guan, J.; Jia, Q. Robustness of Coherence in Noisy Scale-Free Networks and Applications to Identification of Influential Spreaders. IEEE Trans Circuits Syst. II Express Briefs 2020, 67, 1274-1278. [CrossRef]

22. Mofifid, O.; Momeni, M.; Mobayen, S.; Fekih, A. A disturbance-observer-based sliding mode control for the robust synchronization of uncertain delayed chaotic systems: Application to data security. IEEE Access 2021, 9, 16546-16555. [CrossRef]

23. Kang, H.; Liu, K.; Fu, X. Dynamics of an epidemic model with quarantine on scale-free networks. Phys. Lett. A 2017, 381, 3945-3951. [CrossRef]

24. Mei, G.; Wu, X.; Wang, Y.; Hu, M.; Lu, J.A.; Chen, G. Compressive-sensing based structure identifification for multi-layer networks. IEEE Trans. Cybern. 2018, 48, 754-764. [CrossRef] [PubMed]

25. Huang, C.; Zhu, L. Robust evaluation method of communication network based on the combination of complex network and big data. Neural Comput. Appl. 2021, 33, 887-896. [CrossRef]

26. Chutani, M.; Tadic, B.; Gupte, N. Hysteresis and synchronization processes of Kuramoto oscillators on high-dimensional simplicial complexes with the competing simplex-encoded couplings. Phys. Rev. E 2021, 104, 034206. [CrossRef]

27. Pecora, M.; Carroll, L. Master Stability Functions for Synchronized Coupled Systems. Phys. Rev. Lett. 1998, 80, 2109-2112. [CrossRef]

28. Aguirre, J.; Sevilla-Escoboza, R.; Gutíerrez, R.; Papo, D.; Buldú, J. Synchronization of Interconnected Networks: The Role of Connector Nodes. Phys. Rev. Lett. 2014, 112, 248701. [CrossRef]

29. Zhu, J.; Huang, D.; Jiang, H.; Bian, J.; Yu, Z. Synchronizability of multi-layer variable coupling windmill-type networks. Mathematics 2021, 9, 2721. [CrossRef]

30. Deng, Y.; Jia, Z.; Deng, G.; Zhang, Q. Eigenvalue spectrum and synchronizability of multiplex chain networks. Phys. A Stat. Mech. Its Appl. 2020, 537, 122631. [CrossRef]

31. Li, N.; Zheng, W. Bipartite synchronization for inertia memristor-based neural networks on coopetition networks. Neural Netw. 2020, 124, 39-49. [CrossRef] [PubMed]

32. Tang, L.; Wu, X.; Lü, J.; Lu, J.; D'Souza, R. Master stability functions for complete, intralayer, and interlayer synchronization in multiplex networks of coupled Rössler oscillators. Phys. Rev. E 2019, 99, 012304. [CrossRef] [PubMed]

33. Jäntschi, L. The Eigenproblem Translated for Alignment of Molecules. Symmetry 2019, 11, 1027.

34. Barik, S.; Sahoo, G. On the Laplacian spectra of some variants of corona. Linear Algebra Its Appl. 2017, 512, 32-47. [CrossRef]

35. Cvetkovic, D.; Rowlinson, P.; Simic, S. An Introduction to the Theory of Graph Spectra; Cambridge University Press: Cambridge, UK, 2010.

36. Li, X.; Li, X.; Hu, C. Some new results on stability and synchronization for delayed inertial neural networks based on non-reduced order method. Neural Netw. 2017, 96, 91-100. [CrossRef] 